\title{
HPCDb: an integrated database of pancreatic cancer
}

Yonggang Tan ${ }^{1}$, Yongqiang $\operatorname{Tan}^{2}$, Lin $\mathrm{Lu}^{2}$, Heying Zhang ${ }^{1}$, Cheng Sun ${ }^{1}$, Yusi Liang ${ }^{1}$, Juan Zeng ${ }^{1}$, Xianghong Yang ${ }^{3}$, Dan $\mathrm{Li}^{3}$, Huawei Zou ${ }^{1}$

1 Department of Oncology, Shengjing Hospital, China Medical University, 110004

2 Department of Radiology, Columbia University Medical Center, 630 West 168th Street, New York, NY, 10032

3 Department of Pathology, Shengjing Hospital, China Medical University, 110004

Corresponding author: Yonggang Tan. Tel: +86 18940259897; Fax: +86 83955292; Email: tanyg@sj-hospital.org

\begin{abstract}
We have established a database of Human Pancreatic Cancer (HPCDb) through effectively mining, extracting, analyzing, and integrating PC-related genes, single-nucleotide polymorphisms (SNPs), and microRNAs (miRNAs), now available online at http://www.pancancer.org/. Data were extracted from established databases, $\geq 5$ published literature (PubMed), and microarray chips (screening of differentially expressed genes using limma package in $\mathrm{R}, \mid \log _{2}$ fold change $(\mathrm{FC}) \mid>1$ ). Further, protein-protein interactions (PPIs) were investigated through the Human Protein Reference Database. miRNA-target relationships were also identified using the online software TargetScan. Currently, HPCDb contains 3284 genes, 120 miRNAs, 589 SNPs, 10,139 PPIs, and 3904 miRNA-target pairs. The detailed information on PC-related genes (e.g., gene identifier (ID), symbol, synonyms, full name, chip sets, expression alteration, PubMed ID, and PPIs), miRNAs (e.g., accession number,
\end{abstract}


chromosome location, related disease, PubMed ID, and miRNA-target interactions), and SNPs (e.g., SNP ID, allele, gene, PubMed ID, chromosome location, and disease) is presented through user-friendly query interfaces or convenient links to NCBI GEO, NCBI PubMed, NCBI Gene, NCBI dbSNP, and miRBase. Overall, HPCDb provides biologists with relevant information on human PC-related molecules at multiple levels, helping to generate new hypotheses or identify candidate markers.

Keywords: Pancreatic cancer, Single-nucleotide polymorphism; microRNA; Differentially expressed gene; Database construction

As the fourth leading cause of cancer-related death and the fifth most aggressive malignancy (Nagpal et al. 2014), pancreatic cancer (PC) is estimated to cause 227,000 deaths per year globally, and its incidence and mortality rate have been gradually rising (Vincent et al. 2011). Risk factors for PC include environmental factors (e.g., insecticides, chlorinated hydrocarbon solvents, nickel, and gasoline and related compounds), medical/surgical factors (e.g., cirrhosis, chronic pancreatitis, and diabetes), genetic factors, age, sex, and lifestyle (e.g., smoking and diet) (Vincent et al. 2011). As the symptoms of PC are nonspecific, including jaundice, fatigue, loss of appetite and weight, glucose intolerance, pain in the abdomen and back, diarrhea, dizziness, chills, and muscle spasms, its early diagnosis mainly depends on biomarkers (Nolen et al. 2014). Despite substantial progress in the detection and clinical treatment of $\mathrm{PC}$, only approximately $6 \%$ of patients are alive 5 years after 
diagnosis (Siegel et al. 2014). Moreover, PC responds poorly to chemotherapy or radiotherapy, requiring a clearer understanding of the biological mechanisms of PC and identification of its novel biomarkers (Vincent et al. 2011).

During the past decades, basic molecular experiments and high-throughput omics studies have been widely conducted to investigate the molecular mechanisms of PC, and substantial progress has been made. However, the obtained data are too scattered across the literature to be useful to biologists and pharmacologists working on PC. Moreover, some of the data derived from high-throughput omics studies have not been annotated correctly or completely. To overcome these obstacles, a few databases of PC have been established, but they exhibit both advantages and disadvantages. Pancreatic Expression Database (PED, http://www.pancreasexpression.org) is a major component of the European Union project MolDiag-Paca (Cutts et al. 2011a), which focuses mainly on pancreatic-derived omics data (e.g., genomics, transcriptomics, proteomics, and microRNAs (miRNAs)), annotations, and clinical data. Pancreatic Cancer Database (PCD, http://pancreaticcancerdatabase.org/) is a recently established database, which emphasizes literature mining for experimentally demonstrated quantitative alterations of miRNAs, mRNAs, and proteins (Thomas et al. 2014). Pancreatic Cancer Gene Database (PC-GDB, http://www.bioinformatics.org/pcgdb/) mainly provides information on genes involved in PC. Pancreatic Cancer Methylation Database (PCMDB, http://crdd.osdd.net/raghava/pcmdb/) is dedicated to the manual collection and compilation of the methylation status of genes in PC from the published literature (Nagpal et al. 2014). However, in these databases, there is little 
focus on PC-related single-nucleotide polymorphisms (SNPs), miRNA-target relationships, and protein-protein interactions (PPIs), which are supposed to play critical roles in cancer progression (Erichsen and Chanock 2004; Zhu et al. 2013; Sun et al. 2014).

SNPs occur every 1,000-2,000 bases (on average) in human chromosomes (Wang et al. 1998), representing the most widespread type of sequence variation in the genome (Fareed and Afzal 2013). Missense SNPs that fall within coding regions of genes may change the amino acid sequence of the corresponding proteins, while SNPs that fall within non-coding regions of genes may affect the sequence of non-coding RNA, transcription factor binding, mRNA degradation, or gene splicing, influencing individual responses to the environment, risk of disease, and prognosis (Sachidanandam et al. 2001). Thus, SNPs are valuable genetic markers for disease prevention, detection, and cure. SNPs of various genes, such as NR5A2, HNF1A, DPYD, SERPINA3, and ABCG2, have been identified to be associated with PC (Petersen et al. 2010; Pierce and Ahsan 2011; Zeng et al. 2011). Hence, SNP data are an important resource for PC research. Consisting of approximately 22 nucleotides, miRNAs have been reported to regulate protein-coding genes endogenously at the post-transcriptional level (Bartel 2004). Evidence has indicated that miRNAs play critical roles in PC progression through regulating their target genes, and they can serve as bio-markers for PC diagnosis, targets for clinical therapies, and criteria in prognosis prediction (Giovannetti et al. 2010; Ali et al. 2011; Srivastava et al. 2011; Liu et al. 2012; Li et al. 2013). Hence, miRNA-target data are an important resource 
for PC research as well. In addition, investigation of PPIs can provide novel targets for clinical therapies (Agharkar et al. 2011), suggesting the need to compile PPI information of PC-related genes.

In this study, to facilitate the systematic study of PC, HPCDb (Database for Pancreatic Cancer, http://www.pancancer.org/) was constructed through effective mining, extracting, analyzing, and integrating PC-related data of genes, SNPs, miRNAs, PPIs, and miRNA-target relationships.

\section{RESULTS}

HPCDb provides a search engine to survey detailed information on PC-related genes, miRNAs, and SNPs, whose identifier (ID) and/or symbol could serve as query keywords. A rough flowchart of a query is presented in Figure 1. 


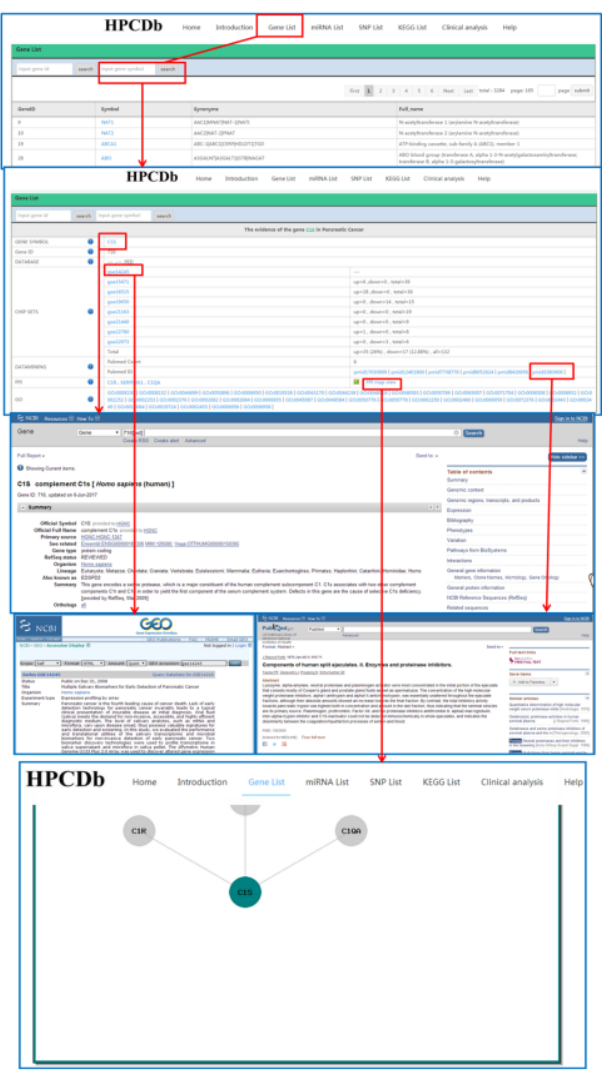

A

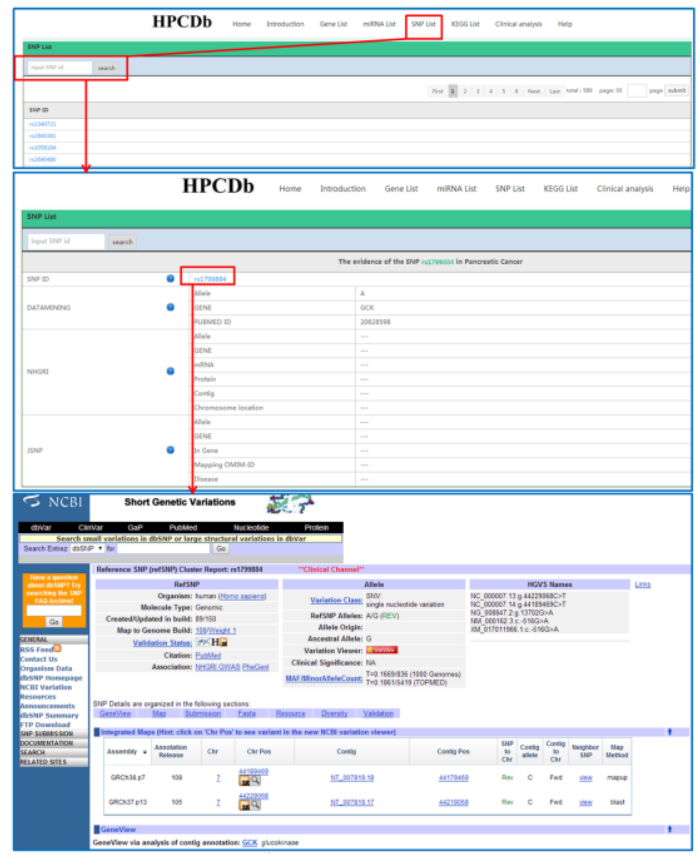

C

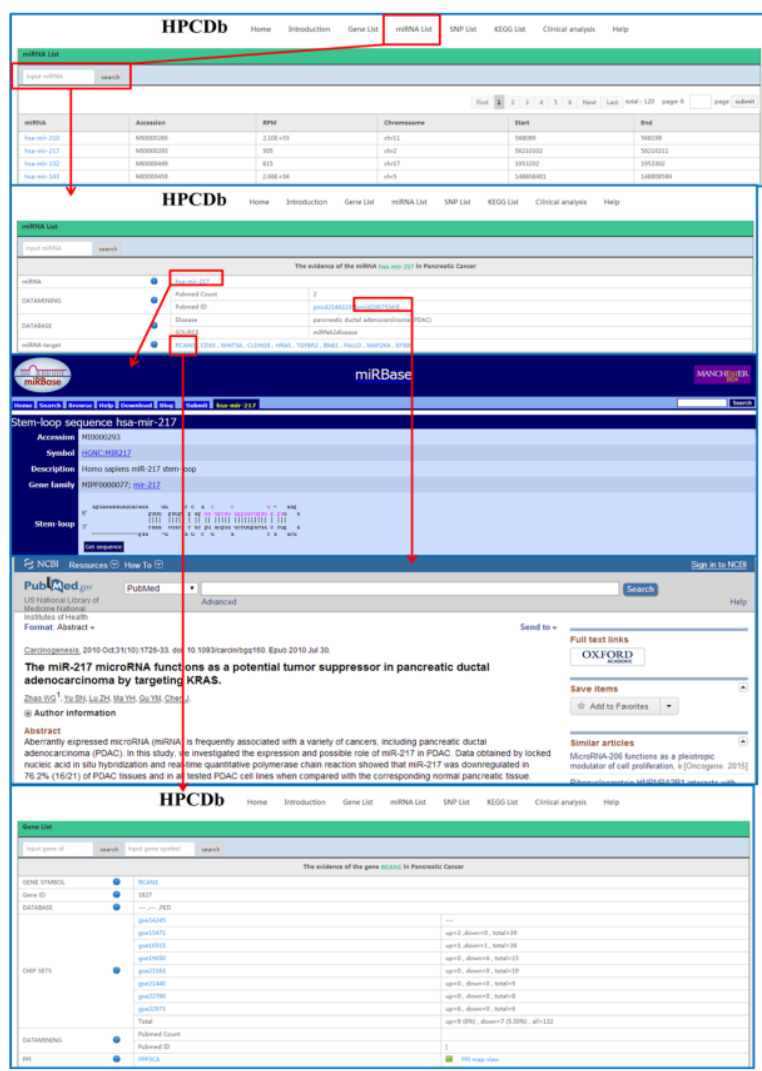

B

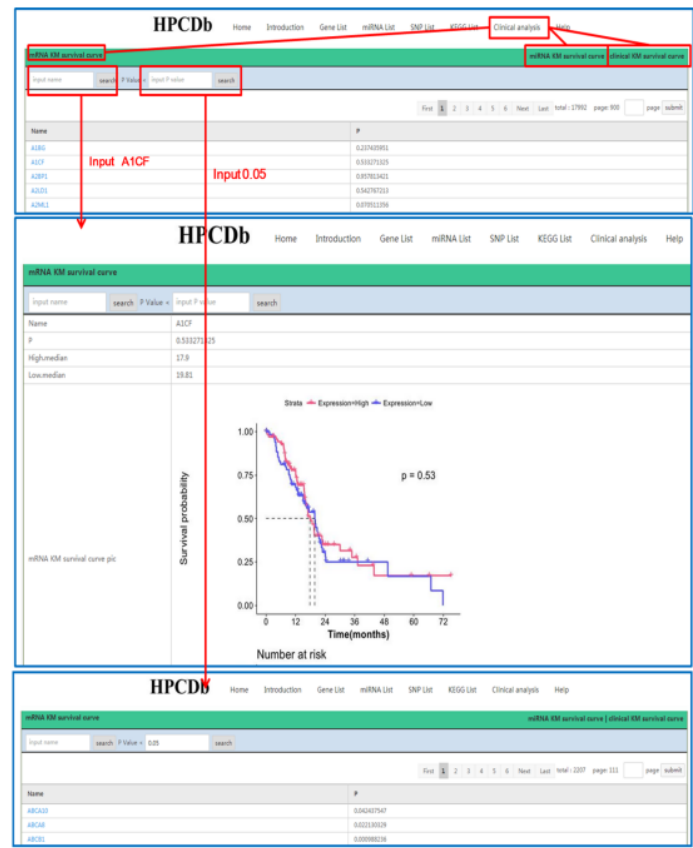

D

\section{Figure 1. Flow charts of a query}

A: A flowchart of a query on the Gene List page. B: A flowchart of a query on the miRNA List page. C: A flowchart of a query on the SNP List page. Red box and arrow: click or tap a search term and then browse; purple box and text: text in purple illustrates the term in the purple box. D: A flowchart of a query on the Clinical Analysis page. 
On the "Gene List" page, PC-related genes are listed alphabetically. After submission of a gene ID or symbol, gene-centered information is displayed on a new page, including gene ID, symbol, synonyms, full name, databases featuring the present gene (PC-GDB, Ensembl, and/or PED), expression alterations based on microarray data, PubMed IDs of literature concerning the present gene, and PPIs. Upon clicking on the gene name, a new page will be displayed, where in the "chip sets" section, the word "total" demonstrates the total numbers of patients who have shown upregulation and/or downregulation of the particular gene. A further click on terms in blue will provide the link to the pages with the corresponding information in the NCBI Gene, NCBI GEO, NCBI PubMed, or HPCDb database, describing the corresponding terms in detail. A click on "PPI map view" will display a new page that illustrates the PPI network of the particular gene and the genes with which it interacts.

On the "miRNA List" page, PC-related miRNAs are also listed alphabetically. After submission of an miRNA ID or symbol, miRNA-centered information will be displayed on a new page, including miRNA ID, accession number in miRBase, reads per million (RPM, a value demonstrating the expression level of miRNA), location (chromosome, start site, and end site), results of data mining (PubMed ID and literature count), as well as databases featuring the present miRNA, disease, source, and miRNA-target relationships. A click on miRNA ID in blue will further link to a new page in miRBase that describes the corresponding miRNA in detail, and a further click on the target genes in blue will subsequently link to new pages in HPCDb that describe the corresponding target genes in detail. 
On the "SNP List" page, PC-related SNPs are listed alphabetically as well. After submission of SNP ID, SNP-centered information will be displayed on a new page, including SNP ID, results of data mining (allele, gene, PubMed IDs), and databases featuring the present SNP (NHGRI GWAS Catalog and/or JSNP databases). A click on SNP ID in blue will further link to a new page in NCBI dbSNP that describes the corresponding SNP in detail.

On the "clinical analysis" page, three categories, namely, mRNA survival curve, miRNA survival curve, and clinical survival curve, are included. Taking mRNA as an example, there were 17,992 mRNA KM survival curves. On the one hand, after input of the gene name, the survival curve of the related genes can be searched. For example, after inputting $\mathrm{ABCB} 1$, the relationship between $\mathrm{ABCB} 1$ expression level and survival rate can be obtained, and $\mathrm{p}=0.00099$ is displayed, indicating a significant correlation between the expression level of $\mathrm{ABCB} 1$ and the survival time. On the other hand, a p-value threshold can instead be input for the search. For example, a total of 2,207 results can be obtained after selecting $p<0.05$. The instructions for the miRNA survival curve and clinical survival curve are the same as the instructions for the mRNA survival curve.

\section{Discussion}

PC is a cancer with extremely high incidence and mortality rate, for which no effective clinical therapy is currently available. To provide a platform for the comprehensive study of this disease, we established HPCDb, a database for 
PC-related genes, miRNAs, SNPs, and clinical analyses, through effectively mining, extracting, analyzing, integrating, and annotating the existing data scattered across various databases and the literature. Information on PC-related genes (e.g., gene ID, symbol, synonyms, full name, chip sets, expression alteration, PubMed ID, and PPIs), miRNAs (e.g., accession, RPM, chromosome location, related disease, PubMed ID, and miRNA-target interactions), SNPs (e.g., SNP ID, allele, gene, PubMed ID, chromosome location, and disease), and clinical analysis was collected and integrated into this system. Currently, the database contains 3284 genes, 120 miRNAs, 589 SNPs, 10,139 PPIs, 3904 miRNA-target pairs, and 17,992 mRNA KM survival curves, as well as 457 miNRA KM survival curves, which are presented through user-friendly query interfaces. Thus, investigators can rapidly investigate whether a gene, miRNA, or SNP is involved in PC progression, and get access to the details associated with these factors.

Compared with previous databases of PC (Cutts et al. 2011b; Nagpal et al. 2014; Thomas et al. 2014), HPCDb has several salient features. (1) Based on the microarray data downloaded from the NCBI GEO database, PC-related DEGs were rescreened, and their changes in expression in association with this disease were calculated and summarized, providing comprehensive data on the possibility of such changes. (2) Various sources were utilized, including omics data, established databases, and literature in PubMed. (3) In addition to PC-related genes and miRNAs, PC-related SNPs, PPIs between PC-related genes, and miRNA-target relationships were investigated as well, expanding the systematic understanding of PC progression. (4) 
Clinical analysis was performed, and prognostic analysis results of PC were visualized. (5) All of the effective information about a search term is displayed on the same page, providing easy access to the molecular information at a glance. (6) Convenient links to NCBI GEO, NCBI PubMed, NCBI Gene, NCBI dbSNP, and miRBase are provided.

Overall, as a freely available web-based resource, $\mathrm{HPCDb}$ provides biologists and pharmacologists with relevant information on human PC-related molecules at multiple levels, helping to generate new hypotheses or identify candidate markers.

\section{MATERIALS AND METHODS}

\section{Database construction}

Relevant data were extracted from established databases, published literature, and microarray chips. The collected data were then analyzed, integrated, and annotated, as shown in Figure 2. 


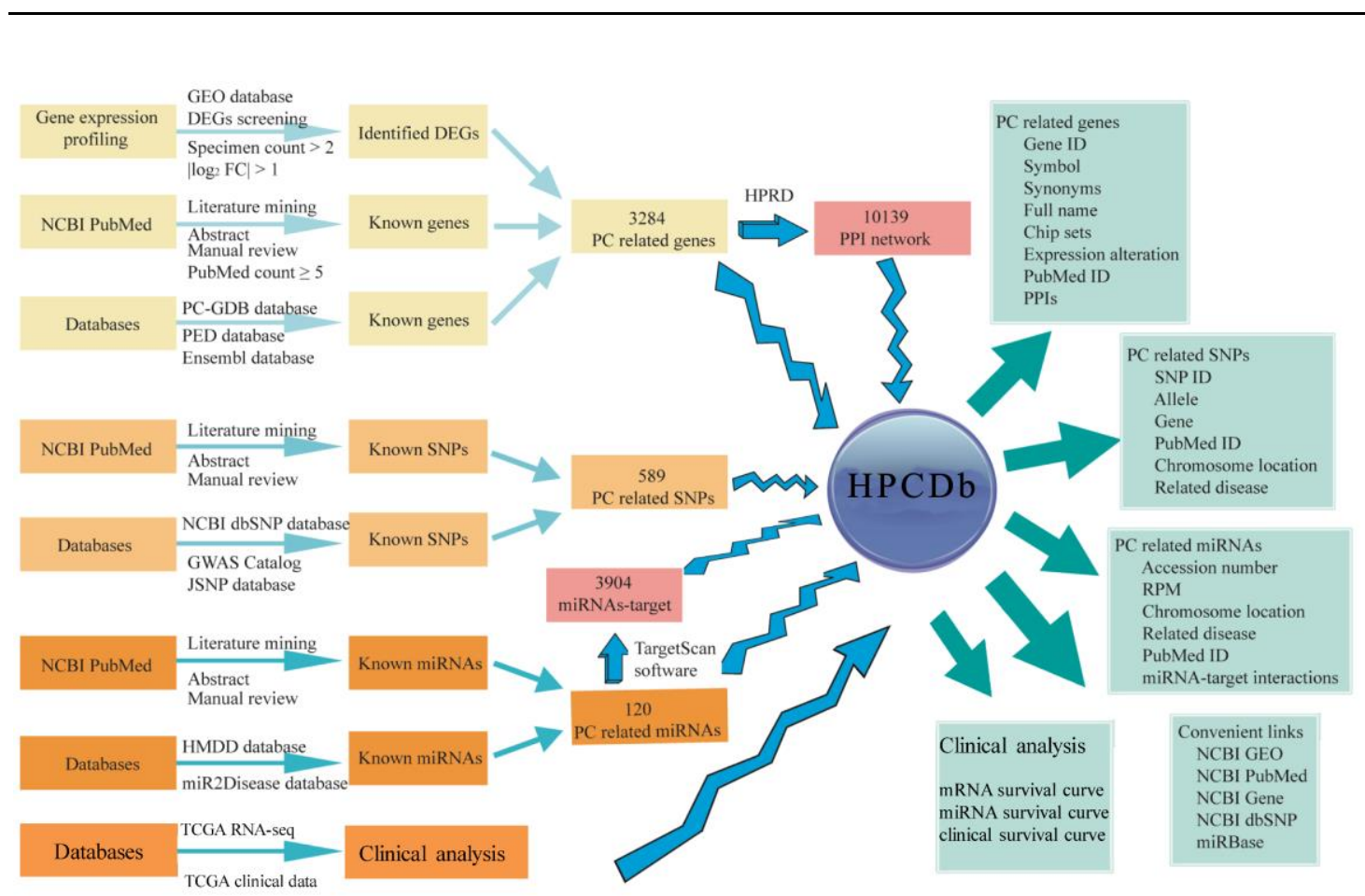

Figure 2. The procedure of database construction

GEO: Gene Expression Omnibus; FC: fold change; NCBI: National Center for Biotechnology Information; PC-GDB: Pancreatic Cancer Gene Database; PED: Pancreatic Expression Database; GWAS: Genome-Wide Association Studies; JSNP: Japanese Single Nucleotide Polymorphisms; HMDD: Human MicroRNA Disease Database; HPRD: Human Protein Reference Database; ID: identifier; RPM: reads per million; DEG: differentially expressed gene; PC: pancreatic cancer; PPI: protein-protein interaction; SNP: single-nucleotide polymorphism

\section{$P C$-related genes}

To collect PC-related genes, the following searches were performed: (1) known genes were extracted from PC-GDB, PED, and Ensembl (http://www.ensembl.org), which store comparative genomics, variation, and regulatory data (Flicek et al. 2012); (2) known genes with PubMed count $\geq 5$ were collected; (3) gene expression profiling datasets containing samples from both a PC group (specimen count $>2$ ) and a healthy control group were downloaded from the National Center for Biotechnology Information $(\mathrm{NCBI})$ Gene Expression Omnibus

(GEO, 
http://www.ncbi.nlm.nih.gov/geo/) database. Specifically, for each downloaded microarray dataset, raw probe-level data were preprocessed based on the corresponding platform and log-scale robust multi-array analysis (McCall et al. 2010), to conduct background correction, missing values estimation, $\log _{2}$ transformation, and value normalization. Moreover, probes without a corresponding gene symbol were omitted, and the expression values of multiple probes mapping to the same gene symbol were averaged as the gene expression value. Subsequently, the limma package in R (Smyth 2005) was utilized to identify genes that were significantly differentially expressed between PC and control groups. Only the genes with $\mid \log _{2}$ fold change (FC) $\mid>1$ were defined as differentially expressed genes (DEGs), and they were also considered as PC-related genes for this database.

In total, 119 and 114 PC-related genes were extracted from PC-GDB and Ensembl, respectively, and 512 DEGs were obtained from PED. After literature mining, 1170 PC-related genes (PubMed count $\geq 5$ ) were obtained. After analyzing gene expression data of microarray chips, 1768 significant DEGs $\left(\left|\log _{2} \mathrm{FC}\right|>1\right)$ were identified between the PC and control groups (Supplementary file 1). After integrating these genes, 3284 PC-related genes were finally obtained (Supplementary file 2). For these genes, Gene Ontology (GO) and Kyoto Encyclopedia of Genes and Genomes (KEGG) annotation analyses were performed to investigate their bio-functions, and the corresponding results are listed in Supplementary file 3. Further, protein-protein interactions (PPIs) of these selected genes were investigated through the Human Protein Reference Database (HPRD, http://www.hprd.org) (Keshava Prasad et al. 
2009), and 10,139 PPIs were obtained (Supplementary file 4). All of the supplemented annotations of selected genes were used for database construction.

\section{$P C$-related miRNAs}

For PC-related miRNAs, the following procedures were performed: (1) known miRNAs were collected from the Human MicroRNA Disease Database (HMDD, http://www.cuilab.cn/hmdd) ( $\mathrm{Lu}$ et al. 2008) and miR2Disease database (http://www.mir2disease.org/) (Jiang et al. 2009); and (2) known PC-related miRNAs were extracted from the literature in PubMed.

In total, 102 PC-related miRNAs were obtained from HMDD and the miR2Disease databases, whereas 38 miRNAs were obtained through literature mining. Conjunctively, 120 PC-related miRNAs were identified. Then, these miRNAs were analyzed with the online software TargetScan to predict their targets (Lewis et al. 2005; Garcia et al. 2011), from which 3904 miRNA-target pairs were identified (Supplementary file 5).

\section{PC-related SNPS}

For PC-related SNPs, we utilized the following search methods: (1) known SNPs were collected from the NCBI Single Nucleotide Polymorphism database (dbSNP, http://www.ncbi.nlm.nih.gov/SNP) (Sherry et al. 2001); the National Human Genome Research Institute (NHGRI), Published Genome-Wide Association Studies (GWAS) Catalog (http://www.genome.gov/), which includes 1751 manually curated 
publications of 11,912 SNPs (Welter et al. 2014); and the Japanese Single Nucleotide Polymorphisms database (JSNP database, http://snp.ims.u-tokyo.ac.jp/) (Hirakawa et al. 2002); and (2) known PC-related SNPs were extracted from the literature in PubMed.

Information on SNP ID (dbSNP database), risk allele, gene symbol, mRNA, and protein was collected and standardized. A total of 346, 21, and 433 PC-related SNPs were screened from NCBI dbSNP, the NHGRI GWAS Catalog, and the JSNP databases, respectively, while 150 SNPs were extracted from more than 500 published reports (Supplementary file 6). Conjunctively, 589 PC-related SNPs were identified.

\section{$P C$-related clinical analysis}

For PC-related clinical analysis, the RNA-seq and miRNA-seq level 3 data of PC in the TCGA database (https://cancergenome.nih.gov/) and PC clinical data were downloaded. Survival curve analysis for PC was performed according to the expression level of mRNAs and miRNAs as well as demographic and clinical characteristics (age, sex, and grade). Survival analysis was conducted for 17,992 mRNAs and 457 miNRAs, and 2207 mRNAs and 29 miRNAs were found to affect the survival time of PC significantly $(\mathrm{p}<0.05)$. Survival analysis for age, sex, and grade found that these three clinical indices did not significantly affect the survival time of PC.

\section{Acknowledgments}


We thank Prof. Fengping Shan and Yuan Yuan for critical advices and fruitful discussions of the article. We also thank Wei Song and his team for technical assistance.

Author contributions: Yonggang Tan, Yoangqiang Tan and Lin Lu conceived and designed the program. Cheng Sun, Juan Zeng and Yusi Liang performed data searching, screening, downloading and standardized processing. Heying Zhang and Dan Li performed Differential gene screening, functional analysis and Text mining analysis of these genes. Yonggang Tan and Xianghong Yang performed Up-and-downstream analysis of pancreatic cancer related genes, including miRNA-target and Protein-Protein interaction. Yongqiang Tan and Huawei Zou performed survival and prognosis analysis of genes, Yoangqiang Tan and Lin $\mathrm{Lu}$ conducted the pancreatic cancer database and website. Yonggang Tan, Yoangqiang Tan and Lin Lu wrote the paper with help from all authors

Conflict of interest statement. None declared.

\section{References}

Agharkar PA, Ethirajan M, Liao J, Yemma M, Magis A, Cance W, Pandey R, Kurenova E. 2011. Abstract C124: Development of novel FAK inhibitors targeting the FAK-VEGFR3 protein-protein interaction site. Molecular Cancer Therapeutics 10: C124.

Ali S, Almhanna K, Chen W, Philip PA, Sarkar FH. 2011. Differentially expressed miRNAs in the plasma may provide a molecular signature for aggressive pancreatic cancer. American journal of translational research 3: 28.

Bartel DP. 2004. MicroRNAs: genomics, biogenesis, mechanism, and function. cell 116: 281-297.

Cutts RJ, Gadaleta E, Hahn SA, Crnogorac-Jurcevic T, Lemoine NR, Chelala C. 2011a. The Pancreatic Expression database: 2011 update. Nucleic acids research 39: D1023-1028.

Cutts RJ, Gadaleta E, Hahn SA, Crnogorac-Jurcevic T, Lemoine NR, Chelala C. 2011b. The pancreatic expression database: 2011 update. Nucleic acids research 39: D1023-D1028. 
Erichsen HC, Chanock SJ. 2004. SNPs in cancer research and treatment. Br J Cancer 90: 747-751.

Fareed M, Afzal M. 2013. Single nucleotide polymorphism in genome-wide association of human population: A tool for broad spectrum service. Egyptian Journal of Medical Human Genetics 14: $123-134$.

Flicek P, Ahmed I, Amode MR, Barrell D, Beal K, Brent S, Carvalho-Silva D, Clapham P, Coates G, Fairley S. 2012. Ensembl 2013. Nucleic acids research: gks1236.

Garcia DM, Baek D, Shin C, Bell GW, Grimson A, Bartel DP. 2011. Weak seed-pairing stability and high target-site abundance decrease the proficiency of 1sy-6 and other microRNAs. Nat Struct Mol Biol 18: 1139-1146.

Giovannetti E, Funel N, Peters GJ, Del Chiaro M, Erozenci LA, Vasile E, Leon LG, Pollina LE, Groen A, Falcone A. 2010. MicroRNA-21 in pancreatic cancer: correlation with clinical outcome and pharmacologic aspects underlying its role in the modulation of gemcitabine activity. Cancer research 70: 4528-4538.

Hirakawa M, Tanaka T, Hashimoto Y, Kuroda M, Takagi T, Nakamura Y. 2002. JSNP: a database of common gene variations in the Japanese population. Nucleic acids research 30: 158-162.

Jiang Q, Wang Y, Hao Y, Juan L, Teng M, Zhang X, Li M, Wang G, Liu Y. 2009. miR2Disease: a manually curated database for microRNA deregulation in human disease. Nucleic acids research 37: D98-104.

Keshava Prasad TS, Goel R, Kandasamy K, Keerthikumar S, Kumar S, Mathivanan S, Telikicherla D, Raju R, Shafreen B, Venugopal A et al. 2009. Human Protein Reference Database--2009 update. Nucleic acids research 37: D767-772.

Lewis BP, Burge CB, Bartel DP. 2005. Conserved seed pairing, often flanked by adenosines, indicates that thousands of human genes are microRNA targets. Cell 120: 15-20.

Li A, Yu J, Kim H, Wolfgang CL, Canto MI, Hruban RH, Goggins M. 2013. MicroRNA array analysis finds elevated serum miR-1290 accurately distinguishes patients with low-stage pancreatic cancer from healthy and disease controls. Clinical Cancer Research 19: 3600-3610.

Liu R, Chen X, Du Y, Yao W, Shen L, Wang C, Hu Z, Zhuang R, Ning G, Zhang C. 2012. Serum microRNA expression profile as a biomarker in the diagnosis and prognosis of pancreatic cancer. Clinical chemistry 58: 610-618.

Lu M, Zhang Q, Deng M, Miao J, Guo Y, Gao W, Cui Q. 2008. An analysis of human microRNA and disease associations. PloS one 3: e3420.

McCall MN, Bolstad BM, Irizarry RA. 2010. Frozen robust multiarray analysis (fRMA). Biostatistics 11: $242-253$.

Nagpal G, Sharma M, Kumar S, Chaudhary K, Gupta S, Gautam A, Raghava GP. 2014. PCMdb: pancreatic cancer methylation database. Sci Rep 4: 4197.

Nolen BM, Brand RE, Prosser D, Velikokhatnaya L, Allen PJ, Zeh HJ, Grizzle WE, Lomakin A, Lokshin AE. 2014. Prediagnostic Serum Biomarkers as Early Detection Tools for Pancreatic Cancer in a Large Prospective Cohort Study. PloS one 9: e94928.

Petersen GM, Amundadottir L, Fuchs CS, Kraft P, Stolzenberg-Solomon RZ, Jacobs KB, Arslan AA, Bueno-de-Mesquita HB, Gallinger S, Gross M et al. 2010. A genome-wide association study identifies pancreatic cancer susceptibility loci on chromosomes 13q22.1, 1q32.1 and 5p15.33. Nat Genet 42: 224-228.

Pierce BL, Ahsan H. 2011. Genome-wide "pleiotropy scan" identifies HNF1A region as a novel pancreatic cancer susceptibility locus. Cancer Res 71: 4352-4358. 
Sachidanandam R, Weissman D, Schmidt SC, Kakol JM. 2001. A map of human genome sequence variation containing 1.42 million single nucleotide polymorphisms. Nature 409: 928-933.

Sherry ST, Ward MH, Kholodov M, Baker J, Phan L, Smigielski EM, Sirotkin K. 2001. dbSNP: the NCBI database of genetic variation. Nucleic acids research 29: 308-311.

Siegel R, Ma J, Zou Z, Jemal A. 2014. Cancer statistics, 2014. CA Cancer J Clin 64: 9-29.

Smyth GK. 2005. limma: linear models for microarray data. In Bioinformatics and computational biology solutions using $R$ and bioconductor, doi:10.1007/0-387-29362-0_23 (ed. R Gentleman, et al.), pp. 397-420. Springer New York.

Srivastava SK, Bhardwaj A, Singh S, Arora S, Wang B, Grizzle WE, Singh AP. 2011. MicroRNA-150 directly targets MUC4 and suppresses growth and malignant behavior of pancreatic cancer cells. Carcinogenesis 32: 1832-1839.

Sun H, Han B, Cheng X, Ma K. 2014. Transcriptional regulatory network and protein-protein interaction to reveal the mechanism of pancreatic cancer. Mol Biol Rep 41: 387-395.

Thomas JK, Kim MS, Balakrishnan L, Nanjappa V, Raju R, Marimuthu A, Radhakrishnan A, Muthusamy B, Khan AA, Sakamuri S et al. 2014. Pancreatic Cancer Database: An integrative resource for pancreatic cancer. Cancer Biol Ther 15: 963-967.

Vincent A, Herman J, Schulick R, Hruban RH, Goggins M. 2011. Pancreatic cancer. Lancet 378: 607-620.

Wang DG, Fan J-B, Siao C-J, Berno A, Young P, Sapolsky R, Ghandour G, Perkins N, Winchester E, Spencer J. 1998. Large-scale identification, mapping, and genotyping of single-nucleotide polymorphisms in the human genome. Science 280: 1077-1082.

Welter D, MacArthur J, Morales J, Burdett T, Hall P, Junkins H, Klemm A, Flicek P, Manolio T, Hindorff L et al. 2014. The NHGRI GWAS Catalog, a curated resource of SNP-trait associations. Nucleic Acids Res 42: D1001-1006.

Zeng H, Yu H, Lu L, Jain D, Kidd MS, Saif MW, Chanock SJ, Hartge P, Risch HA. 2011. Genetic effects and modifiers of radiotherapy and chemotherapy on survival in pancreatic cancer. Pancreas 40: 657-663.

Zhu M, Xu Z, Wang K, Wang N, Li Y. 2013. microRNA and gene networks in human pancreatic cancer. Oncol Lett 6: 1133-1139. 